\title{
Commentary: Long Non-Coding RNA Gene Polymorphisms and Their Expression Levels in Patients With Rheumatoid Arthritis
}

\author{
Jorge Hernández-Bello, Christian Johana Baños-Hernández \\ and José Francisco Muñoz-Valle* \\ Institute for Research in Biomedical Sciences (IICB), University Center for Health Sciences, University of Guadalajara, \\ Guadalajara, Mexico
}

\section{OPEN ACCESS}

Edited by:

Kunihiro Ichinose,

Nagasaki University, Japan

Reviewed by:

Naoki Iwamoto,

Nagasaki University Hospital, Japan Masaru Kato,

Hokkaido University, Japan Agnieszka Paradowska-Gorycka,

National Institute of Geriatrics, Rheumatology and Rehabilitation,

Poland

*Correspondence: José Francisco Muñoz-Valle biologiamolecular@hotmail.com

Specialty section: This article was submitted to Autoimmune and

Autoinflammatory Disorders, a section of the journal

Frontiers in Immunology

Received: 25 October 2021 Accepted: 17 November 2021 Published: 09 December 2021

Citation:

Hernández-Bello J,

Baños-Hernández CJ and Muñoz-Valle JF (2021) Commentary: Long Non-Coding RNA Gene Polymorphisms and Their Expression Levels in Patients With Rheumatoid Arthritis.

Front. Immunol. 12:801266. doi: 10.3389/fimmu.2021.801266
Keywords: ANRIL, Inc-DC, single-nucleotide polymorphism, rheumatoid arthritis, ZFAS1, MALAT1

\section{A Commentary on}

Long Non-Coding RNAs Genes Polymorphisms and Their Expression Levels in Patients With Rheumatoid Arthritis

By Zhang T-P, Zhu B-Q, Tao S-S, Fan Y-G, Li X-M, Pan H-F, et al. (2019). Front. Immunol. 10:2529. doi: $10.3389 /$ fimmu.2019.02529

\section{INTRODUCTION}

We read the paper by Zhang et al. (1) with interest. The authors report on a study that evaluated the association of four long noncoding RNA (lncRNA) (ANRIL, lnc-DC, MALAT1, ZFAS1) gene single-nucleotide polymorphisms (SNPs) with susceptibility to rheumatoid arthritis (RA) patients, as well as their expression levels. They concluded that ANRIL, lnc-DC, MALAT1, and ZFAS1 gene SNPs were not associated with RA susceptibility after false discovery rate (FDR) correction, while altered ANRIL, lnc-DC, MALAT1, and ZFAS1 levels in RA patients suggested that these lncRNAs might play a role in RA.

After carefully reading, we identified some mistakes in the odds ratio (OR) calculations in Table 1 "Genotypes and alleles frequencies of lncRNAs genes polymorphisms in RA patients and normal controls" [sic]. Likewise, there are inconsistencies in the genetic models.

In general, we find that the OR values were calculated and interpreted in an inappropriate way. This is very noticeable when analyzing the frequency of genotypes in cases and controls. For example, for rs1412830, the authors reported an OR $=0.214(0.060-0.761), p=0.017$, for the TT genotype. That OR value suggests that this genotype is a protective or lower risk factor. However, the TT genotype has a higher prevalence in patients than that in controls $(1.97 \%$ vs. $0.42 \%$, respectively); therefore, the OR value should be $>1$.

Regarding genetic models, the authors maintain the same mistake in the OR values. Taking rs1412830 as an example, we once again observed that there is a higher frequency of the TT genotype in patients than that in controls $(1.97 \%$ vs. $0.42 \%$, respectively). However, the authors reported an OR value $=0.211(0.059-0.750), \mathrm{p}=0.016$, but they describe it as a risk factor. We correctly calculate the OR values for your consideration (Table 1). 
TABLE 1 | Correction: Genotypes and allele frequencies of IncRNA gene polymorphisms in RA patients and normal controls.

\begin{tabular}{|c|c|c|c|c|c|c|}
\hline SNP & \multicolumn{2}{|c|}{ Analyze model } & RA (N = 660) n (\%) & Control $(\mathrm{N}=710)$ n (\%) & $p$ value & OR $(95 \% \mathrm{Cl})$ \\
\hline \multicolumn{7}{|l|}{ ANRIL } \\
\hline \multirow[t]{9}{*}{ rs1412830 } & Genotypes & $\Pi$ & $13(1.97)$ & $3(0.42)$ & 0.008 & $4.66(1.32-16.45)$ \\
\hline & & CT & 119 (18.03) & $139(19.58)$ & 0.55 & $0.92(0.70-1.21)$ \\
\hline & & $\mathrm{CC}$ & $528(80.00)$ & $568(80.00)$ & Reference & \\
\hline & Alleles & $\mathrm{T}$ & $145(10.98)$ & $145(10.21)$ & 0.51 & $1.08(0.85-1.38)$ \\
\hline & & C & 1,175 (89.02) & $1,275(89.79)$ & Reference & \\
\hline & Dominant model & $\mathrm{TT}+\mathrm{CT}$ & $132(20.00)$ & $142(20.00)$ & 1 & $1.00(0.76-1.30)$ \\
\hline & & CC & $528(80.00)$ & $568(80.00)$ & Reference & \\
\hline & Recessive model & $\pi$ & $13(1.97)$ & $3(0.42)$ & 0.007 & $4.73(1.29-16.69)$ \\
\hline & & $\mathrm{CC}+\mathrm{CT}$ & 647 (98.03) & 707 (99.58) & Reference & \\
\hline \multirow[t]{9}{*}{ rs944796 } & Genotypes & GG & $11(1.67)$ & $31(4.37)$ & 0.006 & $0.38(0.19-0.78)$ \\
\hline & & GC & 238 (36.06) & 230 (32.39) & 0.28 & $1.13(0.90-1.41)$ \\
\hline & & $\mathrm{CC}$ & $411(62.27)$ & 449 (63.24) & Reference & \\
\hline & Alleles & G & $260(19.70)$ & $292(20.56)$ & 0.57 & $0.95(0.78-1.14)$ \\
\hline & & C & 1,060 (80.30) & $1,128(79.44)$ & Reference & \\
\hline & Dominant model & $\mathrm{GG}+\mathrm{GC}$ & 249 (37.73) & 261 (36.76) & 0.71 & $1.04(0.83-1.30)$ \\
\hline & & $\mathrm{CC}$ & $411(62.27)$ & 449 (63.24) & Reference & \\
\hline & Recessive model & $G G$ & $11(1.67)$ & 31 (4.37) & 0.003 & $0.37(0.18-0.74)$ \\
\hline & & $\mathrm{CC}+\mathrm{GC}$ & 649 (98.33) & 679 (95.63) & Reference & \\
\hline \multirow[t]{9}{*}{ rs61271866 } & Genotypes & AA & 25 (3.79) & $26(3.66)$ & 0.98 & $1.00(0.57-1.76)$ \\
\hline & & TA & 185 (28.03) & $214(30.14$ & 0.39 & $0.9(0.71-1.14)$ \\
\hline & & $\Pi$ & $450(68.18)$ & $470(66.20)$ & Reference & \\
\hline & Alleles & $\mathrm{A}$ & $235(17.80)$ & 266 (18.73) & 0.52 & $0.94(0.77-1.14)$ \\
\hline & & $\mathrm{T}$ & 1,085 (82.20) & $1,154(81.27)$ & Reference & \\
\hline & Dominant model & $\mathrm{AA}+\mathrm{TA}$ & $210(31.82)$ & $240(33.80)$ & 0.43 & $0.91(0.72-1.14)$ \\
\hline & & $\pi$ & $450(68.18)$ & $470(66.20)$ & Reference & \\
\hline & Recessive model & AA & 25 (3.79) & 26 (3.66) & 0.90 & $1.03(0.59-1.81)$ \\
\hline & & $T+\mathrm{TA}$ & 635 (96.21) & $684(96.34)$ & Reference & \\
\hline \multirow[t]{9}{*}{ rs2518723 } & Genotypes & $\pi$ & $111(16.82)$ & 133 (18.73) & 0.26 & $0.83(0.61-1.14)$ \\
\hline & & $\mathrm{CT}$ & 326 (49.39) & 353 (49.72) & 0.53 & $0.92(0.73-1.17)$ \\
\hline & & $\mathrm{CC}$ & 223 (33.79) & $224(31.55)$ & Reference & \\
\hline & Alleles & $\mathrm{T}$ & $548(41.52)$ & 619 (43.59) & 0.27 & $0.91(0.78-1.06)$ \\
\hline & & C & 772 (58.48) & $801(56.41)$ & Reference & \\
\hline & Dominant model & $\mathrm{TT}+\mathrm{CT}$ & 437 (66.21) & $486(68.45)$ & 0.37 & $0.90(0.72-1.13)$ \\
\hline & & $\mathrm{CC}$ & 223 (33.79) & 224 (31.55) & Reference & \\
\hline & Recessive model & $\pi$ & $111(16.82)$ & 133 (18.73) & 0.35 & $0.87(0.66-1.15)$ \\
\hline & & $\mathrm{CC}+\mathrm{CT}$ & 549 (83.18) & 577 (81.27) & Reference & \\
\hline \multirow[t]{9}{*}{ rs3217992 } & Genotypes & $\pi$ & $160(24.24)$ & $152(21.41)$ & 0.11 & $1.27(0.93-1.72)$ \\
\hline & & $\mathrm{CT}$ & $338(51.21)$ & 362 (50.99) & 0.34 & $1.13(0.87-1.45)$ \\
\hline & & $\mathrm{CC}$ & $162(24.55)$ & $196(27.61)$ & Reference & \\
\hline & Alleles & $\mathrm{T}$ & 658 (49.85) & 666 (46.90) & 0.12 & $1.12(0.96-1.30)$ \\
\hline & & $\mathrm{C}$ & $662(50.15)$ & 754 (53.10) & Reference & \\
\hline & Dominant model & $\mathrm{TT}+\mathrm{CT}$ & 498 (75.45) & 514 (72.39) & 0.19 & 1.17 (0.92-1.49) \\
\hline & & $\mathrm{CC}$ & $162(24.55)$ & $196(27.61)$ & Reference & \\
\hline & Recessive model & $\pi$ & $160(24.24)$ & $152(21.41)$ & 0.21 & $1.17(0.91-1.51)$ \\
\hline & & $\mathrm{CC}+\mathrm{CT}$ & $500(75.76)$ & 558 (78.59) & Reference & \\
\hline \multicolumn{7}{|l|}{ Lnc-DC } \\
\hline \multirow[t]{9}{*}{ rs7217280 } & Genotypes & AA & $3(0.45)$ & $4(0.56)$ & 0.74 & $0.78(0.17-3.50)$ \\
\hline & & GA & $52(7.88)$ & $77(10.85)$ & 0.059 & $0.70(0.48-1.01)$ \\
\hline & & $\mathrm{GG}$ & 605 (91.67) & 629 (88.59) & Reference & \\
\hline & Alleles & $A$ & 58 (4.39) & 85 (5.99) & 0.06 & $0.72(0.51-1.01)$ \\
\hline & & $\mathrm{G}$ & 1,262 (95.61) & 1,335 (94.01) & Reference & \\
\hline & Dominant model & $\mathrm{AA}+\mathrm{GA}$ & 55 (8.33) & $81(11.41)$ & 0.057 & $0.70(0.49-1.01)$ \\
\hline & & $\mathrm{GG}$ & 605 (91.67) & 629 (88.59) & Reference & \\
\hline & Recessive model & $\mathrm{AA}$ & $3(0.45)$ & $4(0.56)$ & 0.77 & $0.80(0.18-3.61)$ \\
\hline & & $\mathrm{GG}+\mathrm{GA}$ & 657 (99.55) & 706 (99.44) & Reference & \\
\hline \multirow[t]{7}{*}{ rs10515177 } & Genotypes & GG & $4(0.61)$ & $5(0.70)$ & 0.79 & $0.83(0.22-3.13)$ \\
\hline & & $A G$ & $94(14.24)$ & $117(16.48)$ & 0.24 & $0.84(0.62-1.12)$ \\
\hline & & $\mathrm{AA}$ & $562(85.15)$ & 588 (82.82) & Reference & \\
\hline & Alleles & $G$ & 102 (7.73) & 127 (8.94) & 0.25 & $0.85(0.65-1.11)$ \\
\hline & & $A$ & $1,218(92.27)$ & 1,293 (91.06) & Reference & \\
\hline & Dominant model & $G G+A G$ & $98(14.85)$ & $122(17.18)$ & 0.23 & $0.84(0.62-1.12)$ \\
\hline & & AA & $562(85.15)$ & 588 (82.82) & Reference & \\
\hline
\end{tabular}

(Continued) 
TABLE 1 | Continued

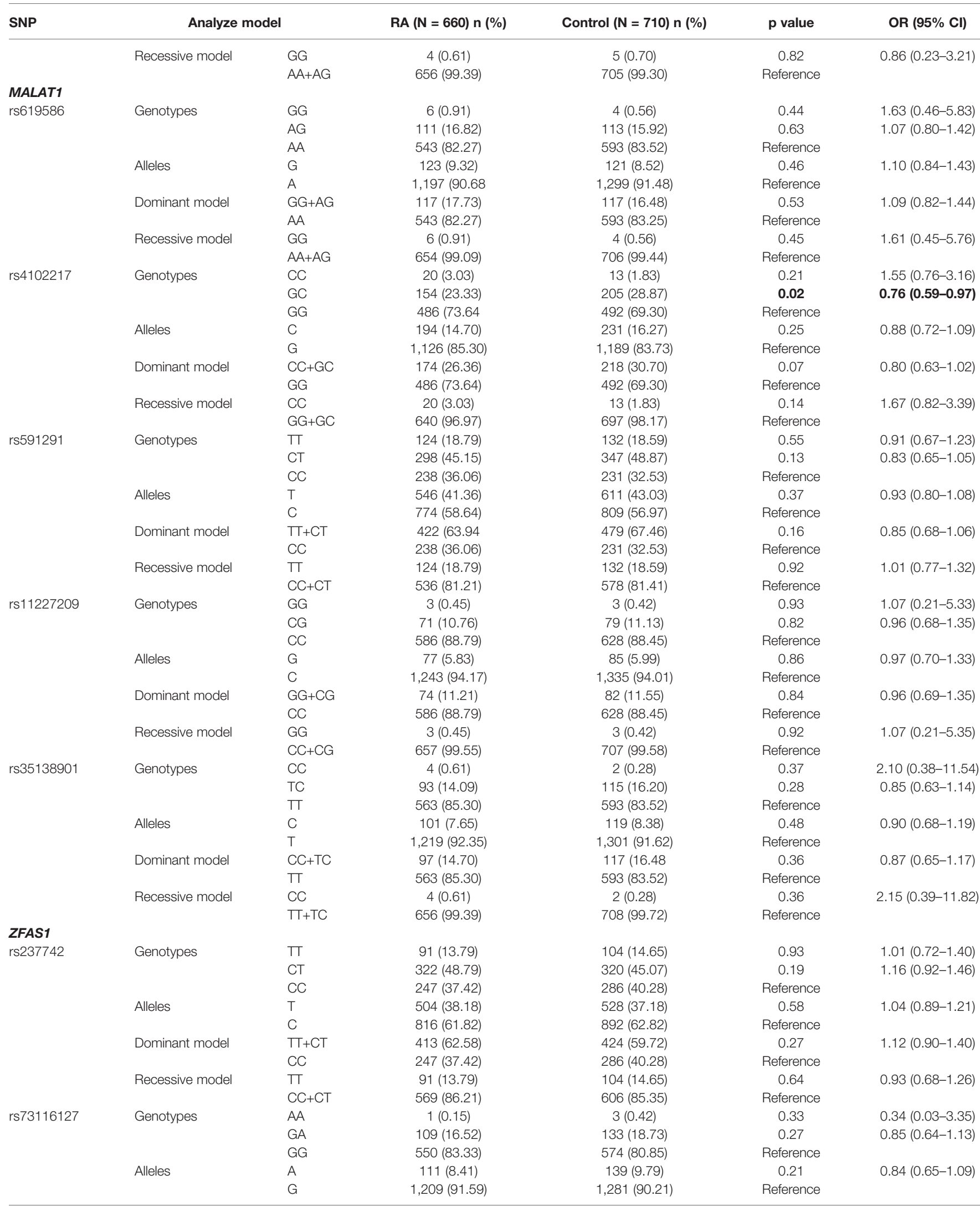


TABLE 1 | Continued

\begin{tabular}{|c|c|c|c|c|c|c|}
\hline SNP & \multicolumn{2}{|c|}{ Analyze model } & $\begin{array}{c}\mathbf{R A}(\mathbf{N}=\mathbf{6 6 0}) \mathbf{n}(\mathbf{\%}) \\
110(16.67)\end{array}$ & $\begin{array}{c}\text { Control }(\mathbf{N}=\mathbf{7 1 0}) \mathbf{n}(\mathbf{\%}) \\
136(19.15)\end{array}$ & $\begin{array}{c}\text { p value } \\
0.23\end{array}$ & $\begin{array}{c}\text { OR }(95 \% \text { Cl) } \\
0.84(0.64-1.11)\end{array}$ \\
\hline & & GG & 550 (83.33) & $574(80.85)$ & Reference & \\
\hline \multirow[t]{7}{*}{ rs6125607 } & Genotypes & $\Pi$ & $74(11.21)$ & $48(6.76)$ & 0.004 & $1.75(1.18-2.60)$ \\
\hline & & CT & $277(41.97)$ & $310(43.66)$ & 0.87 & $1.01(0.81-1.27)$ \\
\hline & & $\mathrm{CC}$ & 309 (46.82) & 352 (49.58) & Reference & \\
\hline & Dominant model & $\Pi+\mathrm{CT}$ & 351 (53.18) & 358 (50.42) & 0.30 & $1.11(0.90-1.38)$ \\
\hline & & $\mathrm{CC}$ & 309 (46.82) & $352(49.58)$ & Reference & \\
\hline & Recessive model & $\pi$ & $74(11.21)$ & $48(6.76)$ & 0.003 & $1.74(1.19-2.54)$ \\
\hline & & $\mathrm{CC}+\mathrm{CT}$ & 586 (88.78) & $662(93.23)$ & Reference & \\
\hline \multirow[t]{5}{*}{ rs6125608 } & Genotypes & $\mathrm{GG}$ & $9(1.36)$ & $11(1.55)$ & 0.70 & $0.84(0.34-2.04)$ \\
\hline & & $A G$ & $125(18.94)$ & $158(22.25)$ & 0.12 & $0.81(0.62-1.05)$ \\
\hline & & $A A$ & $526(79.70)$ & $541(76.20)$ & Reference & \\
\hline & Recessive model & GG & $9(1.36)$ & $11(1.55)$ & 0.77 & $0.87(0.36-2.13)$ \\
\hline & & $A A+A G$ & $651(98.64)$ & $699(98.45)$ & Reference & \\
\hline
\end{tabular}

Bold values denote statistical significance at the $p<0.05$ level.

Cl, confidence interval; InCRNA, long noncoding RNA; OR, odds ratio; RA, rheumatoid arthritis; SNP, single-nucleotide polymorphism.

ANRIL, antisense non-coding RNA in the INK4 locus); Lnc-DC, Lnc-RNA in dendritic cell; MALAT1, metastasis-associated lung adenocarcinoma transcript-1; zinc finger antisense 1.

We recommend that the authors (1) recalculate these data appropriately (2) in order to be able to rediscuss all their results. Also, we suggest that they corroborate the OR values in Table 3 (1), which we could not analyze due to lack of data.

\section{REFERENCES}

1. Zhang T-P, Zhu B-Q, Tao S-S, Fan Y-G, Li X-M, Pan H-F, et al. Long NonCoding RNAs Genes Polymorphisms and Their Expression Levels in Patients With Rheumatoid Arthritis. Front Immunol (2019) 10:2529. doi: 10.3389/ fimmu.2019.02529

2. Sato Y, Suganami H, Hamada C, Yoshimura I, Sakamoto H, Yoshida T, et al. The Confidence Interval of Allelic Odds Ratios Under the Hardy-Weinberg Disequilibrium. J Hum Genet (2006) 51:772-80. doi: 10.1007/s10038-006-0020-6

Conflict of Interest: The authors declare that the research was conducted in the absence of any commercial or financial relationships that could be construed as a potential conflict of interest.

\section{AUTHOR CONTRIBUTIONS}

JH-B wrote this commentary and analyzed the data. CB-H and $\mathrm{JM}-\mathrm{V}$ performed the analysis. All authors contributed to the article and approved the submitted version.

Publisher's Note: All claims expressed in this article are solely those of the authors and do not necessarily represent those of their affiliated organizations, or those of the publisher, the editors and the reviewers. Any product that may be evaluated in this article, or claim that may be made by its manufacturer, is not guaranteed or endorsed by the publisher.

Copyright (c) 2021 Hernández-Bello, Baños-Hernández and Muñoz-Valle. This is an open-access article distributed under the terms of the Creative Commons Attribution License (CC BY). The use, distribution or reproduction in other forums is permitted, provided the original author(s) and the copyright owner(s) are credited and that the original publication in this journal is cited, in accordance with accepted academic practice. No use, distribution or reproduction is permitted which does not comply with these terms. 